Case Report

\section{Carotid artery disease: AngioCT features}

\author{
Joana Ferreira ${ }^{1 *}$, Olinda Miranda1 ${ }^{1}$ Alexandre Lima Carneiro², \\ Sandrina Braga ${ }^{1}$, João Correia Simões ${ }^{1}$, Celso Carrilho ${ }^{1}$, Amílcar \\ Mesquita ${ }^{1}$ and Jorge Cotter ${ }^{1}$ \\ ${ }^{1}$ Hospital da Senhora da Oliveira, Rua dos Cutileiros 114 Creixomil, Guimarães, Portugal \\ ${ }^{2}$ Centro Hospitalar de Trás-os-Montes e Alto Douro, Rua dos Lagoeiros, Portugal
}

\section{Abstract}

The objective of this paper is to emphasis the AngioCT features of carotid dissection/mural hematoma. The image show an internal carotid artery with narrowly eccentric lumen surrounded by a crescent-shaped hypodense mural thickening, with a visibly enhancing vessel wall. The carotid hematoma is a hypodense mural thickening that leads to expansion of the arterial wall, compression of the lumen and release of thrombogenic factors by intimal damage. Hematoma between the intima and media causes vessel expansion diameter and a narrow eccentric lumen. Peripheral hyper density is due to the contrast enhancement of the vasa vasorum in the adventitial layer. The physician should be familiar with the imagiologic features of carotid arterial disease, due to the diferent treatment options.

\section{More Information}

*Address for Correspondence: Joana Ferreira, Hospital da Senhora da Oliveira, Rua dos Cutileiros 114 Creixomil, Guimarães, Portugal, Tel: +351962958421; Email: joana222@gmail.com

Submitted: 16 August 2019 Approved: 24 August 2019 Published: 26 August 2019

How to cite this article: Ferreira J, Miranda O, Carneiro AL, Braga S, Simões JC, et al. Carotid artery disease: AngioCT features. Arch Surg Clin Res. 2019; 3: 059-060.

DOI: dx.doi.org/10.29328/journal.ascr.1001035

Copyright: (c) 2019 Ferreira J, et al. This is an open access article distributed under the Creative Commons Attribution License, which permits unrestricted use, distribution, and reproduction in any medium, provided the original work is properly cited

Keywords: Carotid artery; Dissection; Mural hematoma; Atherosclerosis; AngioCT; Stenosis

\section{Case Report}

A 49-year-old hypertensive female was admitted with left hemiparesis. She had no history of diabetes, dislipidemia, smoking, trauma or cervical pain. Her AngioCT scan showed acute ischemic infarction of the right temporal lobe and severe stenosis at the origin of the right internal carotid artery (Figure 1). The origin of the internal carotid artery had narrowly eccentric lumen surrounded by a crescent-shaped mural thickening, which was less dense than the surround structures and had a visibly enhancing vessel wall (Figure 2) [1]. This aspect is specific of mural hematoma.

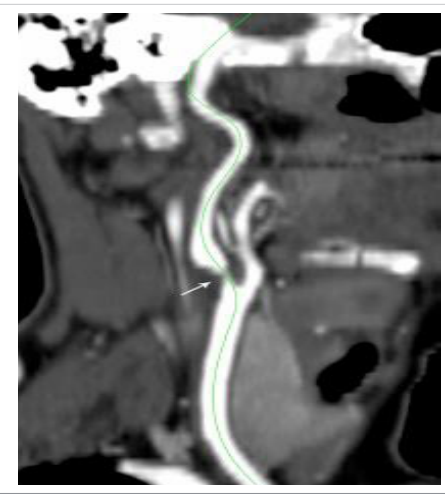

Figure 1: Axial TC image of the right internal carotid artery hematoma. The internal carotid artery has a narrowly eccentric lumen (arrow), resemble a atherosclerotic stenosis.

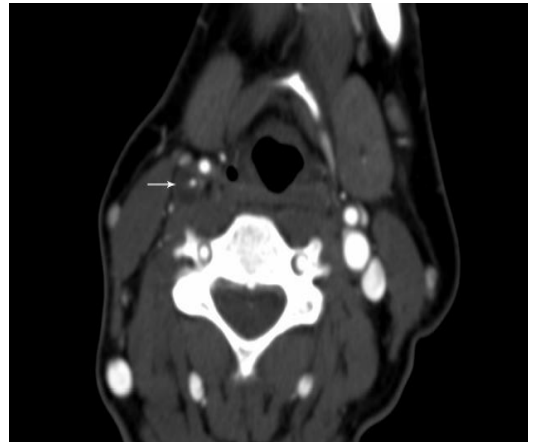

Figure 2: Coronal TC image of the right internal carotid artery hematoma. In this image is clear that the carotid lumen is surrounded by a hypo dense mural thickening, from the hematoma (arrow). The hematoma between the intima and media and causes the vessel diameter expansion. The enhancing vessel wall is visible due to contrast enhancement of vasa vasorum in the adventitial layer.

Systemic intravenous thrombolysis was initiated, but interrupted due to thrombolytic anaphylaxis. The patient was placed on antiaggregation, with a progressive and complete reversion of the left hemiparesis after rehabilitation.

What are the imagiologic features of a carotid dissection? How can we differentiate it from a carotid atherosclerotic stenosis?

\section{Discussion}

Carotid mural hematoma can result from vasa vasorum 
rupture or from intimal tear- dissection. The carotid mural hematoma can be identified on the CT scan as a hypodense mural thickening as is visible in this patient (Figure 2). The hematoma between the intima and media leads to expansion of the arterial wall, compression of the lumen and release of thrombogenic factors by intimal damage, promoting local thrombus formation, as reported in this case [1-3]. The hematoma between the intima and media also results in expansion of the vessel diameter and causes a narrow eccentric lumen as it is possible to recognize in figure 2. Alternatively, hematoma between the media and adventitia may lead to aneurysmal dilatation [3,4]. Peripheral enhancement detected on imaging is due to the contrast enhancement of the vasa vasorum in the adventitial layer and is considered a imagiologic characteristic of carotid dissection (Figure 2) [1,2]. Atherosclerotic plaques are usually localized at the bulb, can be heterogeneous or calcified, show no enhancement of the adventitia and do not alter the diameter of the carotid [5]. Other atherosclerotic plaques are very often seen, sometimes contralaterally at the same site. Sometimes atherosclerotic plaque also has ulceration, defined as plaque depression of $2 \mathrm{~mm}$ deep filled with contrast material, which is a predictor of intra plaque haemorrhage [6].

Internal carotid mural hematoma and dissection can occur spontaneously or associated with trauma, sometimes even minor [1,3,4]. Infections (mainly respiratory), arterial anomalies (such as fibromuscular dysplasia, aortic-root dilatation, bicuspid aortic valve) and inheritable connective tissue disorders have been implicated in the etiology $[1,2,4]$. The treatment options are thrombolysis, antiplatelet or anticoagulation therapy [4]. Endovascular treatment (angioplasty or placement of a self-expanding stent) is not routinely performed [1]. It can be recommended if symptoms persist despite adequate medical management, if antithrombotic therapy is contraindicated, or when there is evidence of an expanding or dissecting aneurysm and cerebral flow is significantly limited [1-3]. Surgical treatment is limited to recurrent ischemia despite medical treatment due to persistent stenosis or aneurysm and has been replaced by endovascular techniques $[1,3,4]$.

On CT scan the carotid mural hematoma has specific characteristics. The carotid wall is thickness, hypo dense with a peripheral enhancement. The vessel diameter is increased. Recognizing the imagiologic characteristics of carotid dissection and differentiate it from carotid atherosclerosis is mandatory, due to the different treatment options.
The patient in the case report does not have any imagiologic evidence of atherosclerotic plaques neither diabetes, dyslipidaemia nor smoking habits.

\section{Acknowledgements}

1. Joana Ferreira one of the patient's physician, made the literature revision and wrote the article.

2. Olinda Miranda one of the patient's physician, helped in the collection of the clinical data, read and corrected the article.

3. Alexandre Lima Carneiro the radiologist, who helped in the image interpretation and in the article writing.

4. Sandrina Braga and João Correia Simões read and corrected the article.

5. Celso Carrilho helped in the diagnosis, read and corrected the article.

6. Amílcar Mesquita the head of vascular surgery department, made the final article correction.

7. Jorge Cotter the head of internal medicine department, made the final article correction.

\section{References}

1. Patel RR, Adam R, Maldjian C, Lincoln CM, Yuen A, et al. Cervical Carotid Artery Dissection: Current Review of Diagnosis and Treatment. Cardiol Rev. 2012; 20: 145-152.

PubMed: https://www.ncbi.nlm.nih.gov/pubmed/22301716

2. Rodallec MH, Marteau V, Gerber S, Desmottes L, Zins M. Craniocervical Arterial Dissection: Spectrum of Imaging Findings and Differential Diagnosis. Radiographics. 2008; 28: 1711-1728.

PubMed: https://www.ncbi.nlm.nih.gov/pubmed/18936031

3. Vilela P, Goulão A. Cervical and intracranial arterial dissection: review of the acute clinical presentation and imaging of 48 cases. Acta Med Port. 2003; 16: 155-164.

PubMed: https://www.ncbi.nlm.nih.gov/pubmed/12868394

4. Thanvi B, Munshi SK, Dawson SL, Robinson TG. Carotid and Vertebral Artery Dissection Syndromes. Postgrad Med J. 2005; 81: 383-388. PubMed: https://www.ncbi.nIm.nih.gov/pubmed/15937204

5. Selwaness M, Bouwhuijsen Q, Onkelen R, Hofman A, Franco O, et al. Atherosclerotic Plaque in the Left Carotid Artery Is More Vulnerable Than in the Right. Stroke. 2014; 45: 3226-3230. PubMed: https://www.ncbi.nlm.nih.gov/pubmed/25228259

6. U-King-Im JM, Fox AJ, Aviv RI, Howard $P$, Yeung $R$, et al. Characterization of Carotid Plaque Hemorrhage. a CT Angiography and MR Intraplaque Hemorrhage Study. Stroke. 2010; 41: 1623-1629. PubMed: https://www.ncbi.nlm.nih.gov/pubmed/20576955 